\title{
El ingreso de los pacientes con accidente cerebro-vascular a una unidad de "stroke" mejora su pronóstico
}

Stroke-unit care improves outcomes of acute stroke patients

Candelise L y Cols. The Lancet. 2007 Jan 27; 369: 299-305.

\section{Objetivo}

Determinar si hay diferencia a los dos años, en la mortalidad y la discapacidad en pacientes con accidente cerebro-vascular (ACV o "stroke" en inglés) que ingresan a una unidad especializada de "stroke" (US) versus los que ingresan a una sala general (SG).

\section{Diseño}

Estudio observacional prospectivo controlado.

\section{Lugar}

260 hospitales de Italia.

\section{Pacientes}

11572 pacientes ingresados durante las primeras 48 horas posteriores a un ACV isquémico o hemorrágico. El $95 \% \mathrm{com}$ pletó el seguimiento a dos años; 4764 pacientes provinieron de la US y 6461 controles de la SG.

\section{Medición de resultados principales}

Se definió US a una sala del hospital que dedicara al menos el $80 \%$ de sus camas a admitir pacientes con ACV y que tuviera al menos un médico y una enfermera especializados. La SG se definió como aquella que no tuviese camas específicamente designadas o no contara con personal entrenado en la atención de esta patología. El diagnóstico de ACV fue corroborado por 16 médicos internistas independientes al estudio, y los puntos finales fueron mortalidad intra y extrahospitalaria y discapacidad posterior al evento, evaluada esta última mediante la escala validada de Rankin, que oscila entre un punto (sin discapacidad) y cinco (discapacidad severa).

\section{Resultados principales}

Los pacientes ingresados a la US tuvieron mejor pronóstico que los controles. Ver tabla 1.

Tabla 1: mortalidad y discapacidad a los dos años luego de accidente cerebrovascular.

\begin{tabular}{l|c|c|c} 
& $\begin{array}{c}\text { Unidad de } \\
\text { "stroke" }\end{array}$ & $\begin{array}{c}\text { Sala general } \\
\text { (control) }\end{array}$ & $\begin{array}{c}\text { Odds ratio } \\
\text { (IC95\%) }\end{array}$ \\
\hline Mortalidad intrahospitalaria & $542(11 \%)$ & $1034(15 \%)$ & $0,78(0,64$ a 0,95$)$ \\
\hline Mortalidad extrahospitalaria & $1363(28 \%)$ & $2382(36 \%)$ & $0,79(0,68$ a 0,91$)$ \\
\hline Muerte o discapacidad & $2611(53 \%)$ & $4112(62 \%)$ & $0,81(0,72$ a 0,91$)$ \\
\hline No retorno al hogar & $1743(35 \%)$ & $2829(3 \%)$ & $0,85(0,74$ a 0,97$)$ \\
\hline
\end{tabular}

${ }^{a}$ Ajustado por edad, sexo, tiempo de arribo, tipo de stroke, presencia de fibrilación auricular, grado neurológico; y estratificado por el tipo de Hospital (universitario o no y número de médicos de planta por sala).

De las 19 variables evaluadas de localización, organización y procesos en la US; solamente la disponibilidad de RMN durante las 24 horas durante los siete días a la semana mostró una discreta asociación con la mortalidad o discapacidad al final del seguimiento, siempre a favor de US. Los pacientes admitidos en la SG tuvieron mejor resultado si estuvieron en salas de neurología pertenecientes a un Hospital Universitario.

\section{Conclusiones}

La probabilidad de muerte o secuela a los dos años luego de un ACV fue menor en los pacientes que ingresaron durante las primeras 48 horas de ocurrido el evento a una unidad especializada que en los ingresados a una la sala general.

Palabras clave: accidente vascular cerebral-pronóstico-unidades de terapia intensiva. Key words: stroke, intensive care units.

Fuente de financiamiento: Beca de la Universidad de Milán, Italia.

\section{Comentario}

El estudio comentado reafirma los resultados de la revisión Cochrane que incluyó más de 5000 pacientes con ACV de 23 estudios aleatorizados. Luego de un seguimiento con una mediana de uno año e independientemente del subtipo de evento ${ }^{1}$, esta revisión había demostrado una reducción del riesgo de muerte global (OR 0,86; IC95\% 0,71 a 0,94) del riesgo de mortalidad o cuidado institucional (OR 0,80; IC95\% 0,71 a 0,90 ) y del de mortalidad o dependencia (OR 0,78; IC95\% 0,68 a 0,89 ) en los pacientes que habían sido ingresados a unidades de Stroke.

En 1986, Tage Strand reportó el primer estudio prospectivo sobre 193 pacientes en el cual, independientemente de la edad y la etiología del $\mathrm{ACV}^{2}$, se apreció una mejor evolución y una menor estadía hospitalaria en los ingresados a la US. De esta forma, se redimensionó el tratamiento de los pacientes con $A C V$, en quienes, desde el nihilismo terapéutico o la estrategia de "esperar y ver" se pasó al tratamiento intensivo y a la atención por expertos de enfermería y médicos, impactando en el resultado de estos pacientes. Surge entonces la controversia sobre la necesidad de un lugar específico para el tratamiento, ya que algunos expertos argumentaban que solamente el accionar de un equipo de "stroke", entendido como un grupo de médicos expertos en el tratamiento de estos pacientes, era suficiente para obtener los mismos beneficios, independientemente del área física donde se internaran los pacientes. Sin embargo, a través de un estudio aleatorizado que incluyó 457 pacientes, Andrew Evans y col. inclinaron la balanza a favor de la US, especialmente en los pacientes con ACV isquémico moderado $^{3}$. Respecto a su costo-efectividad, en una cohorte prospectiva que reclutó 395 participantes, Moodie y col. reportaron que la US es más costo-efectiva que la $\mathrm{SG}^{4}$

Conclusiones del comentador

El ingreso temprano -antes de las 48 horas de producido el ACV- a una US se asocia a claros beneficios pronósticos, independientemente del tipo de evento y de las características de los pacientes o las instituciones.

Luis Camputaro [ FCCM FAHA. Director del Programa de Neurointensivismo. Servicio de Terapia Intensiva de Adultos. Hospital Italiano de Buenos Aires. luis.camputaro@ hospitalitaliano.org.ar ]

Camputaro Luis. El ingreso de los pacientes con accidente cerebro-vascular a una unidad de "stroke" mejora su pronóstico. Evid. actual. práct. ambul; 10(6): 166, Nov-Dic.2007. Comentado de Candelise L y col. Stroke-unit care for acute stroke patient: an observational follow-up study. The Lancet. 2007 Jan 27;369:299-305. PMID: 17258670

\section{Referencia}

1. Strok Unit Trialist Collaboration, Organized inpatient (stroke unit) care for stroke. Cochrane Database Syst Rev $2001 ; 3:$ CD000197.

2. Strand T et al. Stroke Unit Care - Who Benefits? 377 Comparisons With General Medical Care in Relation to Prognostic Indicators on Admission. Stroke 1986. $17: 377-381$

3. Evans A, et al. Randomized Controlled Study of Stroke Unit Care Versus Stroke Team Care in Different Stroke Subtypes. Stroke 2002.33:449-455.

4. Moodie M, et al, for the SCOPES Study Group. Stroke. 2006. 37:2790-2795. 